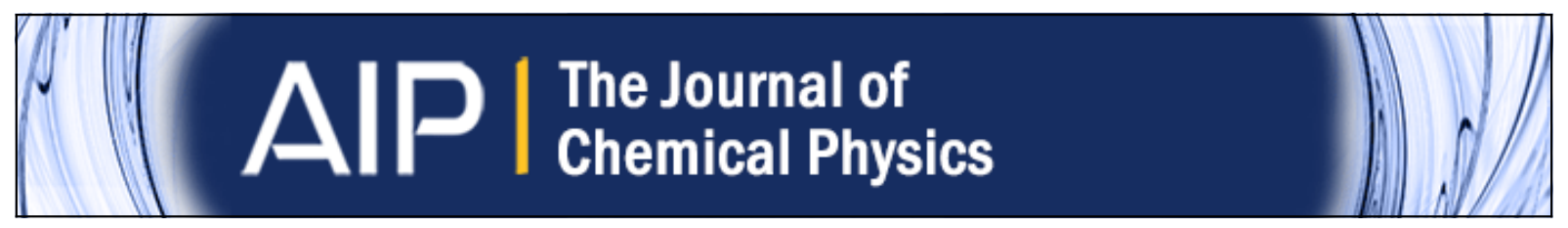

Diffusive spatio-temporal noise in a first-passage time model for intracellular calcium release

Mark B. Flegg, Sten Rüdiger, and Radek Erban

Citation: The Journal of Chemical Physics 138, 154103 (2013); doi: 10.1063/1.4796417

View online: http://dx.doi.org/10.1063/1.4796417

View Table of Contents: http://scitation.aip.org/content/aip/journal/jcp/138/15?ver=pdfcov

Published by the AIP Publishing

Alp Re-register for Table of Content Alerts 


\title{
Diffusive spatio-temporal noise in a first-passage time model for intracellular calcium release
}

\author{
Mark B. Flegg, ${ }^{1, \text { a) }}$ Sten Rüdiger, ${ }^{2}$ and Radek Erban ${ }^{1, b)}$ \\ ${ }^{1}$ Mathematical Institute, University of Oxford, 24-29 St Giles', Oxford OX1 3LB, United Kingdom \\ ${ }^{2}$ Institut für Physik, Humboldt-Universität zu Berlin, 12489 Berlin, Germany
}

(Received 23 November 2012; accepted 8 March 2013; published online 17 April 2013)

\begin{abstract}
The intracellular release of calcium from the endoplasmic reticulum is controlled by ion channels. The resulting calcium signals exhibit a rich spatio-temporal signature, which originates at least partly from microscopic fluctuations. While stochasticity in the gating transition of ion channels has been incorporated into many models, the distribution of calcium is usually described by deterministic reaction-diffusion equations. Here we test the validity of the latter modeling approach by using two different models to calculate the frequency of localized calcium signals (calcium puffs) from clustered $\mathrm{IP}_{3}$ receptor channels. The complexity of the full calcium system is here limited to the basic opening mechanism of the ion channels and, in the mathematical reduction simplifies to the calculation of a first passage time. Two models are then studied: (i) a hybrid model, where channel gating is treated stochastically, while calcium concentration is deterministic and (ii) a fully stochastic model with noisy channel gating and Brownian calcium ion motion. The second model utilises the recently developed two-regime method [M. B. Flegg, S. J. Chapman, and R. Erban, "The two-regime method for optimizing stochastic reaction-diffusion simulations," J. R. Soc., Interface 9, 859-868 (2012)] in order to simulate a large domain with precision required only near the $\mathrm{Ca}^{2+}$ absorbing channels. The expected time for a first channel opening that results in a calcium puff event is calculated. It is found that for a large diffusion constant, predictions of the interpuff time are significantly overestimated using the model (i) with a deterministic non-spatial calcium variable. It is thus demonstrated that the presence of diffusive noise in local concentrations of intracellular $\mathrm{Ca}^{2+}$ ions can substantially influence the occurrence of calcium signals. The presented approach and results may also be relevant for other cell-physiological first-passage time problems with small ligand concentration and high cooperativity. (C) 2013 American Institute of Physics. [http://dx.doi.org/10.1063/1.4796417]
\end{abstract}

\section{INTRODUCTION}

The dynamics of intracellular $\mathrm{Ca}^{2+}$ is a major determinant of many cell signaling processes. ${ }^{2}$ In order to address a host of different target processes, cells orchestrate the elevation of $\mathrm{Ca}^{2+}$ concentration by a complex machinery of $\mathrm{Ca}^{2+}$ transport and binding. Early modeling approaches considered deterministic equations based on macroscopic rate equations. ${ }^{3,4}$ Later, it was found that release of $\mathrm{Ca}^{2+}$ from the endoplasmic reticulum (ER) can occur in a localized and highly random manner, ${ }^{5}$ thus rendering the deterministic modeling approach for $\mathrm{Ca}^{2+}$ dynamics incomplete.

In many cell types, release of $\mathrm{Ca}^{2+}$ from the ER occurs through inositol 1,4,5-trisphosphate receptor $\left(\mathrm{IP}_{3} \mathrm{R}\right)$ channels in the ER's membrane. The receptors regulate $\mathrm{Ca}^{2+}$ transport in response to changes of $\mathrm{IP}_{3}$ and $\mathrm{Ca}^{2+}$ concentration mediated by binding sites on the cytosolic side of the ER's membrane. ${ }^{6}$ While increases in the concentration of the second messenger $\mathrm{IP}_{3}$ generally promote release, the dependence on cytosolic $\mathrm{Ca}^{2+}$ concentration is biphasic and mediated by two types of binding sites. Small increases in $\mathrm{Ca}^{2+}$ concentration compared to rest level concentrations increase the open probability of $\mathrm{IP}_{3} \mathrm{R}$ channels. The stimulation $\mathrm{Ca}^{2+}$ binding

\footnotetext{
a)Electronic mail: mark.flegg@maths.ox.ac.uk.

b) Electronic mail: erban@maths.ox.ac.uk.
}

gives rise to a self-amplifying mechanism called $\mathrm{Ca}^{2+}$ induced $\mathrm{Ca}^{2+}$ release (CICR): $\mathrm{Ca}^{2+}$ released by one or several channels diffuses in the cytosol and increases the open probability of neighbouring channels by binding to their stimulatory binding sites. As the level of $\mathrm{Ca}^{2+}$ rises further, inhibitory binding of $\mathrm{Ca}^{2+}$ dominates. Consequently, the open probability decreases significantly as $\mathrm{Ca}^{2+}$ levels reach large values. Taken together, activating and inhibiting binding processes in combination with $\mathrm{Ca}^{2+}$ diffusion allow for a commonly accepted model for cooperative openings and closings of receptor channels. ${ }^{7}$

Elevations of $\mathrm{Ca}^{2+}$ concentrations appear either as quasideterministic waves or as localized events over spatial distances on the order of a micrometer. ${ }^{7}$ In many cells, $\mathrm{IP}_{3} \mathrm{R}$ channels are distributed in clusters on the ER's membrane. It is often found that CICR synchronizes channels within clusters, resulting in events that were identified with the localized patterns often called puffs. ${ }^{8}$ In this regime, $\mathrm{Ca}^{2+}$ release does not spread to neighbouring clusters, which are typically separated by a few micrometers. Recent studies emphasize the role of sub-cellular $\mathrm{Ca}^{2+}$ rises for physiological function. ${ }^{9-11}$

Since noise that originates from the small copy-number of involved ion channels has been recognized as relevant, stochastic modeling has successfully been applied and now can explain many of the aspects of $\mathrm{Ca}^{2+}$ signals. ${ }^{12,13}$ 
However, as the computational cost is too large, the Brownian motion of $\mathrm{Ca}^{2+}$ ions has been excluded in most studies. The most detailed simulations to date treat $\mathrm{Ca}^{2+}$ concentration as well as the concentration of further diffusing species as deterministic quantities. ${ }^{14}$ Because of their spatial localization, clusters of channels have been studied with theoretical methods by considering only a single cluster and ignoring the coupling to channels outside of the cluster. Recently, puffs have also been modeled in a hybrid manner by coupling Markov gating schemes for clustered channels to deterministic equations for local $\mathrm{Ca}^{2+}$ concentration within the cluster microdomain. ${ }^{12,15-17}$

The amplitude of diffusive fluctuations in receptor- $\mathrm{Ca}^{2+}$ binding can be discussed as in the classical theory of concentration sensing in chemoreception. ${ }^{18-20}$ According to this argument, the accuracy in measuring a concentration $c$ by receptors is limited by $\delta c / c=1 / \sqrt{\operatorname{Dca} \tau}$, where $D$ is the diffusion constant of the molecules with concentration $c, a$ is the dimension of the receptor or the receptor cluster, and $\tau$ is the time scale of the relevant binding/unbinding process. Taking typical values of these parameters $\left(D=200 \mu \mathrm{m}^{2} \mathrm{~s}^{-1}\right.$, $a=1 \mu \mathrm{m}, c=0.02 \mu \mathrm{M}$, and $\tau=1 \mathrm{~s})$ one obtains $\delta c / c$ $\approx 0.02$, which suggests that diffusive fluctuations can be safely neglected compared to the average concentration. As we will discuss here, this noise may not always be neglected in the large diffusion limit.

In this paper, we show that stochastic effects (taken into account by the detailed modeling of discrete calcium ions and their Brownian motion) has consequences for the $\mathrm{Ca}^{2+} \mathrm{dy}-$ namics. Simulation of the detailed stochastic model is enabled by a recently developed multiscale approach ${ }^{1}$ which is summarized in Sec. II. We specifically study $\mathrm{Ca}^{2+}$ puffs and the period of their appearance. Puffs have taken a central role in the modeling of intracellular $\mathrm{Ca}^{2+}$ dynamics, since an integrative model of local and global release should incorporate $\mathrm{Ca}^{2+}$ puffs as elementary building blocks of global waves and oscillations. ${ }^{21}$ In this paper, we show that the noise contributes to a decrease in interpuff times. The paper is divided into Secs. II (Methods), III (Results), and IV (Discussion). In Sect. II, we summarize both the mean-field model and the computational method used to simulate the detailed stochastic model.

\section{THEORY AND SIMULATION METHODS}

To simplify our analysis we neglect the binding and unbinding of $\mathrm{IP}_{3}$ as well as the binding to inhibitory binding sites. We consider $\mathrm{IP}_{3} \mathrm{R}$ channels releasing $\mathrm{Ca}^{2+}$ from the ER to the cytosol to consist of $K=4$ identical subunits with each subunit carrying a binding site that together assume the activating role for the channel. The apparent cooperativity in $\mathrm{Ca}^{2+}$ binding requires a minimum number $N$ of calcium ions to be bound for opening of the channels. Although $N=3$ is traditionally used in modelling, ${ }^{4}$ we will also discuss consequences of different values of $N \in\{1,2, \ldots, K\}$. We will study how much time passes until the number of activated subunits reaches $N$ for any of the cluster's channels, given a suitable initial condition on the state of all four binding sites. This first passage time can be regarded as a realistic approximation of the real interpuff interval (IPI) since it has been suggested that that almost every opening of a single channel triggers a puff. ${ }^{8}$ Although we do not simulate the inhibitory dynamics explicitly, we take it into account by careful selection of the initial state to which the channel is reset after each puff. Specifically, we let the number of activated subunits equilibrate to a distribution, constrained by the requirement that the channel is closed (the number of activated subunits is less than $N$ ) but otherwise allowing for all possible numbers of activated subunits to occur at natural relative probabilities.

\section{A. Mean-field non-spatial model and its analysis}

Calcium channels from the ER to the cytosol are usually arranged in closely packed clusters consisting of up to 10 channels. $^{22}$ Here we denote the number of channels as $C$ and use $C=9$ unless otherwise stated. Each channel has $K=4$ subunits. We assume that $\mathrm{IP}_{3}$ concentrations are large. We submit, therefore, that the channel opens and releases $\mathrm{Ca}^{2+}$ in the form of a puff if at least $N=3$ of the four subunits are activated on a single channel in the cluster. A subunit is activated when a $\mathrm{Ca}^{2+}$ ion is bound to it. The $\mathrm{Ca}^{2+}$ ions are also permitted to dissociate themselves from a subunit, thereby deactivating it. This chemical reaction is given by

$$
\mathrm{Ca}^{2+}+\text { inactive subunit } \underset{k_{2}}{\stackrel{k_{1}}{\rightleftarrows}} \text { active subunit, }
$$

where $k_{1}$ is the rate of activation per unit of $\mathrm{Ca}^{2+}$ concentration per inactive subunit measured in units $(\mu \mathrm{Ms})^{-1}$ and $k_{2}$ is the deactivation rate per active subunit measured in units $\mathrm{s}^{-1}$.

Assuming that $\mathrm{Ca}^{2+}$ concentration is large and equal to $c_{0}$, the mean-field approximation of the probability that a particular subunit is active at equilibrium can be estimated as

$$
P_{\mathrm{act}}=\frac{k_{1} c_{0}}{k_{1} c_{0}+k_{2}} \text {. }
$$

We define the state of a channel to be the number of subunits which are active, i.e., the state of each channel is taken from the set $\{0,1,2, \ldots, K\}$. We assume that each subunit is activated and deactivated independently. Cooperativity of calcium dependent activation is taken into account by the requirement that ${ }^{6} N>1$. Assuming that the average $\mathrm{Ca}^{2+}$ concentration in the cytosol remains constant in space and time we can gain an approximation for the probability of a channel to be in state $\{i\}, i=0,1,2, \ldots, K$, at equilibrium in the form of the binomial distribution

$$
P_{i}^{e}=\left(\begin{array}{c}
K \\
i
\end{array}\right)\left(1-P_{\mathrm{act}}\right)^{K-i} P_{\mathrm{act}}^{i} .
$$

We assume that initially the ion channel is closed (states $\{0\},\{1\}, \ldots,\{N-1\})$. The probability that the channel is in state $\{j\}$ at the end of the inhibitory phase of the channel cycle is given by

$$
P(\{j\} \mid\{0,1, \ldots, N-1\})=\frac{P_{j}^{e}}{\sum_{i=0}^{N-1} P_{i}^{e}},
$$

for $j \leq N-1$. Let $\mathbf{P}(t)=\left[P_{0}(t), P_{1}(t), \ldots, P_{N-1}(t)\right]^{T}$ be the vector of probabilities to be in each of the non-open states as 
a function of time. Transition rates between these states are given as follows:

$$
\{0\} \underset{k_{1,0}}{\stackrel{k_{0,1}}{\rightleftarrows}}\{1\} \underset{k_{2,1}}{\stackrel{k_{1,2}}{\rightleftarrows}} \ldots \underset{k_{N-1, N-2}}{\stackrel{k_{N-2, N-1}}{\rightleftarrows}}\{N-1\} \stackrel{k_{N-1, N}}{\longrightarrow} \emptyset,
$$

where $k_{i, i+1}=(K-i) k_{1} c_{0}, k_{i, i-1}=i k_{2}$ and $\emptyset$ denotes the open channel. Solving the corresponding master equation, we can express $\mathbf{P}(t)$ by the matrix exponential

$$
\mathbf{P}(t)=\mathbf{P}(0) \exp [\mathbf{A} t]
$$

where $\mathbf{A}$ is the tridiagonal matrix given by $A_{i, i-1}=k_{i-1, i}$, $A_{i, i}=-\left[k_{i, i+1}+k_{i, i-1}\right]$, and $A_{i, i+1}=k_{i+1, i}$. Estimating the initial probabilities $\mathbf{P}(0)$ in Eq. (6) using (4), we can compute the probability density of the channel opening time by

$$
\bar{f}_{M C}(\bar{\tau})=-\sum_{i=0}^{N-1} \frac{\mathrm{d} P_{i}}{\mathrm{~d} \bar{\tau}}(\bar{\tau}),
$$

where the bar placed over the $\tau$ indicates it is in reference to a single channel rather than the cluster as a whole. Equation (7) is an exact solution to the model described by (5) but this model assumes that there is no noise in the local concentration of calcium ions. In this manuscript, reaction system (5) will be simulated using Monte Carlo simulations which will be used to give $f_{M C}$, since, in reality the distribution (7) has a rather complicated analytical form when written in full. The Monte Carlo simulation methodology is explained in Sec. II B.

A simpler analytical form for the probability density (7) can be approximated if the probability $P_{N-1}(t)$ is small. If $P_{N-1}(t)$ is small, then the rate of transition to the open state from the state $\{N-1\}$ will be slow. The response of the states $\{0\},\{1\}, \ldots,\{N-1\}$ to the absorption by the open state is comparably swift. Subsequently, the system of states remains approximately steady over time with respect to each other (the relative probability to be in each of the states $\{0\},\{1\}, \ldots$, $\{N-1\}$ remains constant in time). This means that each probability $P_{i}(t) \approx P_{i} \exp \left(-P_{N-1} k_{N-1, N} t\right), i=1, \ldots, N-1$, where $P_{i}$ is the initial probability of being in state $\{i\}$. Thus, the distribution $\bar{f}_{T}(\bar{\tau})$ for the opening time $\bar{\tau}$ is given approximately by

$$
\bar{f}_{T}(\bar{\tau}) \approx \bar{r} \exp (-\bar{r} t)
$$

where

$$
\bar{r} \approx k_{N-1, N} P(\{N-1\} \mid\{0,1, \ldots, N-1\}) .
$$

Here $P(\{N-1\} \mid\{0,1, \ldots, N-1\})$ is given by (4). The accuracy of this theoretical approximation (8), specifically compared with the Monte Carlo simulations (which are described by the full solution (7)), will be discussed in Sec. III A. The rate at which puffs are initiated $r$ for this simple approximation is given by the rate at which any of the $C$ channels in the cluster are opened. Since the distribution is assumed to be exponential, the rate at which puffs are initiated from a cluster of $C$ channels is given by

$$
r \approx C \bar{r} .
$$

Using a choice of parameters compatible with experimental puff properties ${ }^{17} k_{1}=100(\mu \mathrm{Ms})^{-1}, k_{2}=20 \mathrm{~s}^{-1}, c_{0}$
$=0.02 \mu \mathrm{M}$ in Eq. (9), we obtain the approximate mean first passage time for the opening of a single channel $\bar{\tau}=\bar{r}^{-1}$ $\approx 6.083 \mathrm{~s}$. According to (10), the interpuff time is $\tau \approx(C \bar{r})^{-1}$ $=0.68 \mathrm{~s}$ for $C=9 . N=3$ has been used for all calculations unless otherwise specified.

\section{B. Monte Carlo simulation of the channel states}

The simple theoretical formula (10) requires the assumption that the opening time of a channel is distributed exponentially. In this paper, we will compare (10) with two types of stochastic simulations. The first one is a Monte Carlo (MC) simulation of $C$ reaction systems (5) for $C$ channels. The MC simulation of the state of $C$ channels assumes steady reaction rates consistent with a constant concentration $c_{0}$. Diffusion (and therefore diffusive noise) is not considered in this simulation and it is therefore the least accurate method of the two numerical techniques. However, it is the most efficient of the two numerical routines. The simulation activates and deactivates subunits from the $C$ channels according to (1) until one channel has $N$ active subunits and the channel opens. The time for this to occur is stored and an approximate mean interpuff time $E(\tau)$ is determined by averaging the simulation time over many realizations. Since this simulation technique is efficient we are able to easily obtain $10^{6}$ simulations giving precise estimates of the distribution and mean of the variable $\tau$ (under the assumption of no fluctuations in concentration $c_{0}$ ).

The MC algorithm initializes the states of the $C$ channels by assigning the state $\{j\}$ independently to each channel according to the probability (4) for being in the state $\{j\}$, given that the channel is closed. Then the Gibson-Bruck algorithm ${ }^{23}$ for simulating $C$ continuous-time Markov chains (given by (5)) is used until one of the channels is in state $\{N\}$.

It is important to note that these MC simulations result in interpuff times that are distributed according to the exact solution to the master equation given by (7). In Sec. III, these MC simulations will be compared specifically to the distribution (8) to highlight differences between the approximate theoretical solution and the full solution to the model in the absence of noise in the local concentration of calcium ions.

\section{Spatial stochastic modelling}

The constant concentration $c_{0}=0.02 \mu \mathrm{M}$ of $\mathrm{Ca}^{2+}$ in the cytosol is relatively small, because it corresponds to $12 \mathrm{Ca}^{2+}$ ions per $1 \mu \mathrm{m}^{3}$. At small concentrations, the frequency of ion interactions with the channels are infrequent and highly noisy. This noise manifests itself in a time fluctuating concentration that is experienced by the channels with a distribution that has a mean of $c_{0}$. To analyse quantitatively how this noise effects the rate $\bar{r}$, one has to consider detailed spatio-temporal stochastic simulations of $\mathrm{Ca}^{2+}$ ions. There are two main methods for the simulation of stochastic reaction-diffusion processes: ${ }^{24}$ off-lattice Brownian dynamics methods ${ }^{25}$ and on-lattice methods. ${ }^{26}$ The former of these methods allows for a greater amount of microscopic detail and is usually preferred in situations where microscopic detail plays a role in the model and/or the concentration of molecules is low. The latter of these methods only accepts 
motion of molecules between lattice points and is therefore limited by the resolution of the lattice. However, the implementation of on-lattice methods tends to be more efficient and so find use when concentrations are large and well-mixed on a microscopic level. Since it is important in the case of $\mathrm{Ca}^{2+}$ ion channels to know where each molecule of $\mathrm{Ca}^{2+}$ reacts with calcium channels and because the concentration of molecules is small, we find it prudent to use an off-lattice Brownian dynamics simulation (at least in the regions close to the channel). In order to improve the efficiency of Brownian dynamics, we use a multiscale approach ${ }^{1}$ introduced in the following Sec. II D.

\section{Two regime method}

Consider the $1 \times 1 \times 1 \mu \mathrm{m}^{3}$ box that sits inside the cytosol such that the cluster of ion channels lies centralized on the bottom facet. Molecules inside this box can be simulated according to Brownian motion. That is, after defining a time step $\Delta t$, the $n$th molecule updates its location according to the formula

$$
\mathbf{X}_{n}(t+\Delta t)=\mathbf{X}_{n}(t)+\sqrt{2 D \Delta t} \boldsymbol{\xi}_{n},
$$

where $D$ is the diffusion constant, $\mathbf{X}_{n}=\left(x_{n}, y_{n}, z_{n}\right)^{T}$ is the three-dimensional location of the molecule and $\boldsymbol{\xi}_{n}$ is a vector of three independent normally distributed random numbers with a mean of zero and unit variance.

To simulate reversible binding (1), we define three parameters ${ }^{27}$ radius of interaction $R=30 \mathrm{~nm}$, dissociation radius $\sigma=R / 2=15 \mathrm{~nm}$ and the reaction rate $\lambda$. At each time step, $\mathrm{Ca}^{2+}$ ions will bind to an empty binding site with a probability $\lambda \Delta t$ whenever their distance from the site is less than ${ }^{28}$ $R$. The choice of $\lambda$ determines the rate of reaction $k_{1}$. The reaction rate $k_{1}$ between empty binding sites and free calcium ions is dependent on $\lambda$ and $R$ but it is also dependent on the dissociation radius $\sigma$. This is because, dissociating ions that are placed too close to the ion channels will likely immediately rebind to a subunit if $\lambda$ is too large. In the paper by Lipkova et al.,${ }^{27} \lambda$ is derived such that in equilibrium the expected flux of molecules into the sphere of radius $R$ is balanced by the rate of dissociation $k_{2}$. In order that $\lambda$ correctly represents the reaction (1), it is given implicitly by the equation

$$
k_{1}=\frac{4 \pi \sigma D\left(R_{\lambda}-\tanh \left(R_{\lambda}\right)\right)}{\tanh \left(R_{\lambda}\right) \cosh \left(R_{\lambda}-\sigma_{\lambda}\right)-\sinh \left(R_{\lambda}-\sigma_{\lambda}\right)},
$$

where $R_{\lambda}=R \sqrt{\lambda /(2 D)}$ and $\sigma_{\lambda}=\sigma \sqrt{\lambda /(2 D)}$. Note the $\sqrt{2}$ on the denominator for $R_{\lambda}$ and $\sigma_{\lambda}$ is due to the fact that volume of interaction around the channels is only half that of the full sphere considered by Lipkova et $a l .{ }^{27}$ because they lie on the boundary of the domain. Each $\mathrm{Ca}^{2+}$ ion that is activating a subunit dissociates and deactivates that subunit with a probability of $k_{2} \Delta t$. The dissociated $\mathrm{Ca}^{2+}$ ion is placed on the sphere of radius $\sigma$ around the channel it was bound to. We set the default diffusion constant to be $D=220 \mu \mathrm{m}^{2} \mathrm{~s}^{-1}$ and set the time step to $\Delta t=R^{2} /(20 D) \mathrm{s}$. The factor of $1 / 20$ was found to be sufficient to satisfy the requirement of Eq. (12) (that is $\sqrt{2 D \Delta t} \ll R)$ such that the numerical reaction rate was within $1 \%$ of the defined reaction rate of $k_{1}=100(\mu \mathrm{M} \mathrm{s})^{-1}$.
A pertinent question that remains is; how do the molecules behave over boundaries? ${ }^{29}$ It might be argued that since the domain is much larger than the cluster of channels, we may consider the domain to be closed from the rest of the cytosol and implement reflective boundary conditions not just on the membrane containing the channels but also those facets that represent the truncation of the cytosol space. This reasoning does not result in a physically realistic simulation. First, as molecules are absorbed by subunits of the ion channels, naturally this creates a void in which molecules are expected to be supplemented by the $\mathrm{Ca}^{2+}$ ions in the cytosol. For example, if 12 molecules are initialized in the $1 \times 1 \times 1 \mu \mathrm{m}^{3}$ domain then over the course of the simulation some of these may be absorbed. If, for example, 4 are absorbed, the effective concentration of free $\mathrm{Ca}^{2+}$ ions in the closed box would no longer be at $c_{0}$. Moreover, reflective boundaries would also not account for fluctuations of the number of molecules inside the box $1 \times 1 \times 1 \mu \mathrm{m}^{3}$ above the ER's membrane. One way to fix this problem would be by increasing the size of the simulated domain. However, increasing the size of the domain from $1 \times 1 \times 1 \mu \mathrm{m}^{3}$ to $L \times L \times L \mu \mathrm{m}^{3}$ increases the expected number of molecules in the domain by a factor of $L^{3}$ which makes Brownian dynamics simulations slower by the factor of $L^{3}$. It is important to note that while we demand a large domain to get the correct amount of noise in the concentration near the channels, microscopic detail of the $\mathrm{Ca}^{2+}$ ions is not of interest sufficiently far from the channels. Therefore, we can apply the two regime method (TRM) which allows for the Brownian dynamics model described here to be used in the region of interest while a more efficient on-lattice model is used in the rest of the computational domain. ${ }^{1}$ We couple, therefore, a small $1 \times 1 \times 1 \mu \mathrm{m}^{3}$ off-lattice model box, the "region of interest," with a larger $5 \times 5 \times 5 \mu \mathrm{m}^{3}$ on-lattice domain consisting of $1 \times 1 \times 1 \mu \mathrm{m}^{3}$ compartments according to the TRM. ${ }^{1}$ A diagram of the TRM model domain is shown in Figure 1.

The increase in computational time of this TRM model compared with that of the closed off-lattice $1 \mu \mathrm{m}^{3}$ domain model by itself was approximately 2 instead of a factor increase of the order of 100 that could be expected if the Brownian dynamics model were to be used over the same domain. The error that is associated with the TRM is to leading order proportional to the spatial derivative of the expected concentration of the diffusing ions at the interface between the "region of interest" and the rest of the domain. Since, for the duration of the simulation, this expected concentration is spatially homogenous, we do not need to worry about the errors that are introduced using the TRM. Therefore, the choice of the size of the "region of interest" was determined to approximately balance computational effort between the region of interest and the rest of the domain.

\section{RESULTS}

Let us define $f_{M C}(\tau), f_{T R M}(\tau)$ and $f_{T}(\tau)$ to represent the probability distribution for the interpuff time $\tau$ that is derived from MC simulations (7), TRM simulations (no analytical description) and the approximate theoretical result given by formulae (8)-(10), respectively, for $C=9$. We use overbars to 


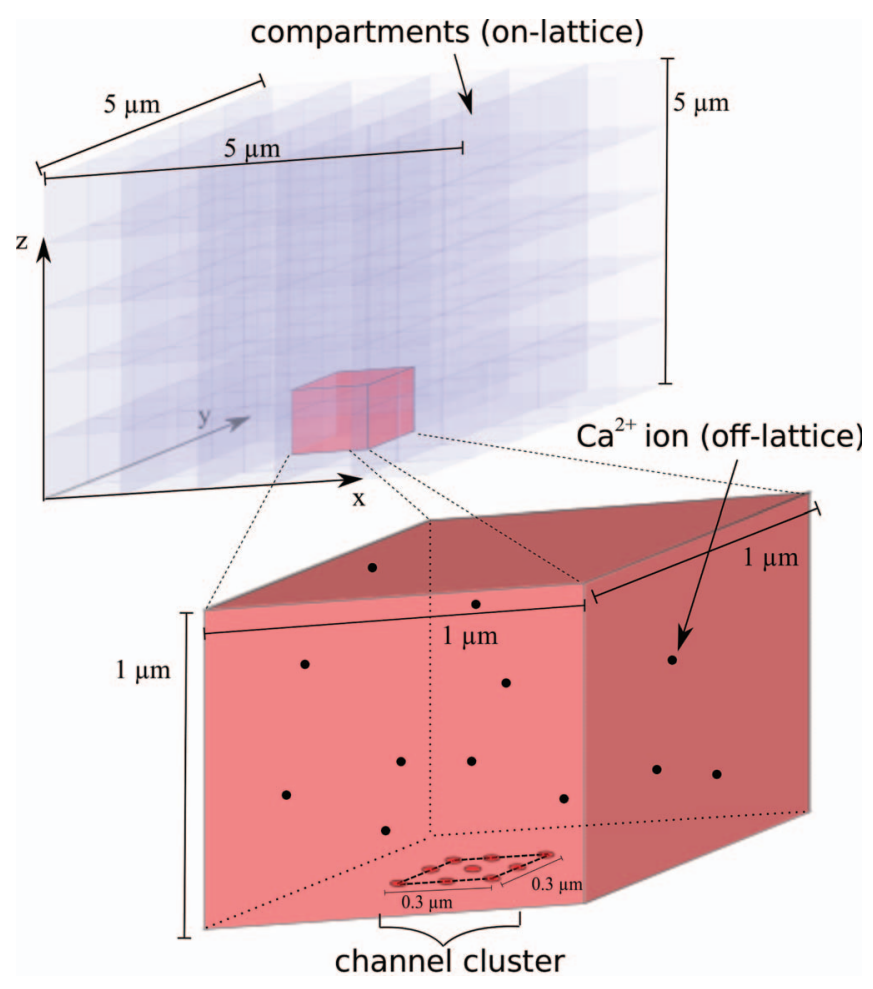

FIG. 1. Representation of the TRM model domain (top panel). Blue boxes model $\mathrm{Ca}^{2+}$ ions on-lattice and the red box models $\mathrm{Ca}^{2+}$ ions off-lattice. The bottom panel shows details of the red box.

indicate the case $C=1$. In Figure 2, we plot the distributions $\bar{f}_{M C}(\bar{\tau}), \bar{f}_{T R M}(\bar{\tau})$, and $\bar{f}_{T}(\bar{\tau})$.

Distributions $\bar{f}_{M C}(\bar{\tau})$ and $\bar{f}_{T R M}(\bar{\tau})$ were determined using the ksdensity function in MATLAB's statistics toolbox. The ksdensity function computes a probability density estimate from a sample. The estimate is based on a normal ker-

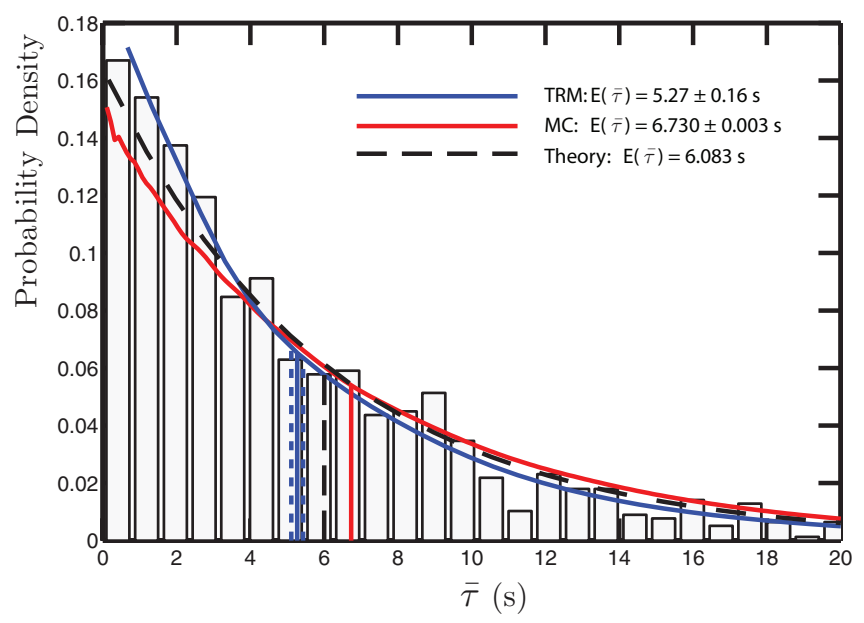

FIG. 2. Probability distributions and means for the interpuff time for $C$ $=1$ given by theoretical estimates $\bar{f}_{T}(\bar{\tau})$ (black dashed line), $10^{6} \mathrm{MC}$ simulations $\bar{f}_{M C}(\bar{\tau})$ (red solid line), and $10^{3}$ TRM simulations $\bar{f}_{T R M}(\bar{\tau})$ (blue solid line). The actual simulated TRM simulation data is indicated by the histogram showing actual simulated probabilities over intervals of time and blue dashed lines indicating the subsequent standard error in the mean. Parameters used: $N=3, k_{1}=100(\mu \mathrm{M} \mathrm{s})^{-1}, k_{2}=20 \mathrm{~s}^{-1}, c_{0}=0.02 \mu \mathrm{M}$, and $D=$ $220 \mu \mathrm{m}^{2} \mathrm{~s}^{-1}$ (for TRM simulations only). The standard deviation of the MC opening time data was $6.732 \mathrm{~s}$ and was $5.717 \mathrm{~s}$ for the TRM opening time data. nel function, using a window parameter ("width") that is a function of the number of points in the sample. The density is evaluated at 100 equally spaced points covering the range of the data in the sample. ${ }^{30}$ In all three cases, the mean is indicated. Non-spatial MC simulations were averaged over $10^{6}$ realizations. The TRM simulations are more computationally intensive than the MC simulations since they have to find the activation and deactivation events by simulating $\mathrm{Ca}^{2+}$ ions in a three-dimensional spatial domain. The number of simulations that could be run for finding expected values of $\tau$ were typically of the order of thousands. One possible way for decreasing the computational demand on the simulations would be to use a modified form of Eqs. (5.6)-(5.8) from the paper from Lipkova et al. ${ }^{27}$ This would save the factor of 20 from the time step in the region of interest $\left(\Delta t=R^{2} / D\right.$ insteady of $\left.\Delta t=R^{2} /(20 D)\right)$. However, this would not affect the computational effort required for the on-lattice domain representing the rest of the cytosolic space. Since the TRM was only run for $10^{3}$ simulations, standard error is therefore indicated along with the mean with dashed blue lines and a histogram representing the actual obtained probabilities within intervals of time is also plotted. For MC simulations and TRM simulations, the standard deviation of the opening time samples are also given. For exponential distributions $\bar{f}_{M C}(\bar{\tau})$ and $\bar{f}_{T R M}(\bar{\tau})$ the standard deviation should be equal to the mean but instead are slightly larger than the mean. This indicates that while these distributions appear to be exponentially distributed (as discussed as an approximation in Sec. II A), this is not strictly the case. Figure 3 shows the comparison in the distributions $f_{M C}(\tau)$ and $f_{T R M}(\tau)$ that result from a cluster of $C$ $=9$ channels. The ratio of expected times $\tau$ does not seem to vary much if the number of channels $C$ is increased and this is because the distributions are close to exponential distributions. This indicates that the ion channels in the spatial TRM

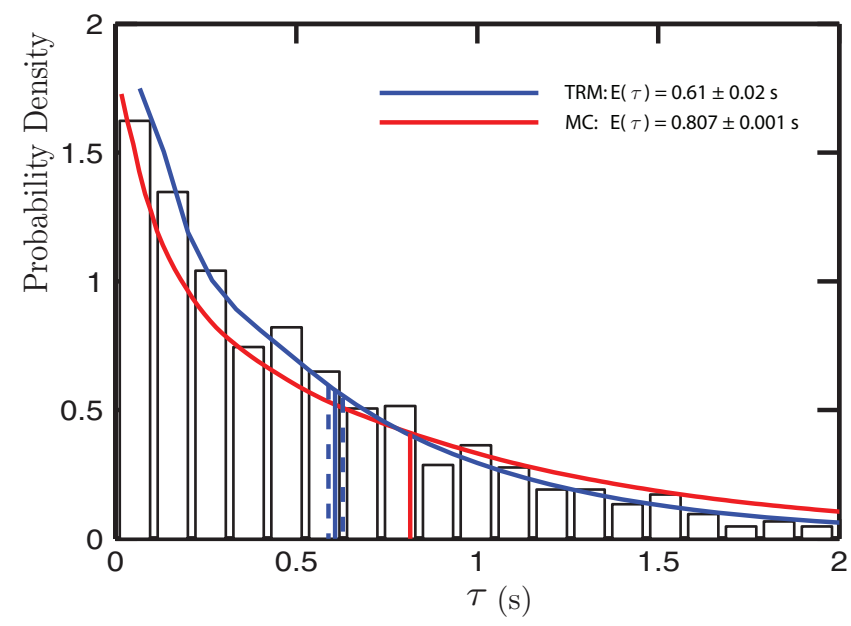

FIG. 3. Probability distributions and means for the interpuff time for $C=9$ given by $10^{6} \mathrm{MC}$ simulations $f_{M C}(\tau)$ (red solid line) and $10^{3}$ TRM simulations $f_{T R M}(\tau)$ (blue solid line). The actual simulated TRM simulation data is indicated by the histogram showing actual simulated probabilities over intervals of time and blue dashed lines indicating the subsequent standard error in the mean. Parameters used: $N=3, k_{1}=100(\mu \mathrm{M} \mathrm{s})^{-1}, k_{2}=20 \mathrm{~s}^{-1}$, $c_{0}=0.02 \mu \mathrm{M}$, and $D=220 \mu \mathrm{m}^{2} \mathrm{~s}^{-1}$ (for TRM simulations only). The standard deviation of the MC opening time data was $0.810 \mathrm{~s}$ and was $0.649 \mathrm{~s}$ for the TRM opening time data. 
simulations seem to exhibit minimal buffering in the cluster. That is, the channels in the cluster tend to work independently for the parameters used in these simulations.

\section{A. Comparison of exponential distribution $f_{T}(\tau)$ with MC simulations $f_{M C}(\tau)$}

The assumptions used to obtain $f_{T}(\tau)$ in (8) relies on states $\{0\},\{1\}, \ldots,\{N-1\}$ having occupation probabilities that are constant with respect to the time given that the channel has not opened. Indeed, knowing the probability for a channel to be in the state $\{N-1\}$ will give the instantaneous rate for the channel to open (i.e., to obtain the state $\varnothing$ in the reaction system (5)). However, the state $\emptyset$ of the channel is an absorbing state and therefore the relative probabilities to be in states $\{0\},\{1\}, \ldots,\{N-1\}$ must change over time, however gradually, according to formula (6) which is reflected in the MC simulation results $f_{M C}(\tau)$ and in (7). Thus, while it is clear that the MC simulation results in $d \bar{f}_{M C}(\bar{\tau}) /\left.d \bar{\tau}\right|_{\bar{\tau}=0}$ $=d \bar{f}_{T}(\bar{\tau}) /\left.d \bar{\tau}\right|_{\bar{\tau}=0}$, it is also expected that the rate for a channel to open is less than the rate predicted by (9) as time progresses. Therefore $d \bar{f}_{M C}(\bar{\tau}) /\left.d \bar{\tau}\right|_{\bar{\tau}>0}>d \bar{f}_{T}(\bar{\tau}) /\left.d \bar{\tau}\right|_{\bar{\tau}>0}$ which results in an expected $\bar{\tau}$ that is larger for the MC simulations than that predicted by (8)-(10). This is in agreement with our MC simulations (see Figure 2). While the approximation (8) predicts that $E(\bar{\tau})=6.083 \mathrm{~s}$, it was found that $1 \times 10^{6}$ nonspatial MC simulations give $E(\bar{\tau})=6.730 \mathrm{~s}$.

\section{B. Comparison of MC simulations $f_{M C}(\tau)$ with TRM simulations $f_{T R M}(\tau)$}

Assuming that the approximate rate for the channels to open is constant in time and approximately equal to that given in (9), it is easy to see that the opening rate as a function of concentration is nonlinear. Figure 4 shows the plot of opening rates versus concentration along side the second derivative of this curve, estimated by (9). At low concentrations of $\mathrm{Ca}^{2+}$, there is a high amplitude noise in the instantaneous local concentration that is experienced by the channels. To analyze the effect of this noise and how it is affected by the diffusion we use the TRM model and compare the results to the non-spatial MC simulations. Initially the noise represents some local concentration that is distributed with a mean of $c_{0}$. The initial instantaneous rate for a channel to open can be considered by (9) given the initial fluctuation in $c_{0}$. This rate is expected to be greater than predicted in (9) and MC simulations for $N>$ 1. This is because for $N>1$, Eq. (9) is a convex function of $c_{0}$ and an increase in the expected rate $\bar{r}$ from that of MC simulations is therefore a consequence of Jensen's inequality. Note that if $N=1$, Eq. (9) is linear with respect to $c_{0}$ and therefore TRM simulation results should be the same as non-spatial MC simulation results. Interestingly, for real $\mathrm{Ca}^{2+}$ ion channels $(N=3)$ the convexity (second derivative) of the opening rate versus concentration has a local maximum near the concentration of $c_{0}=0.02 \mu \mathrm{M}$. This would imply that the biological parameters are near optimal values to emphasize the difference that diffusive noise plays in determining the rate at which the calcium channels will open. Figure 4 indicates that for $N>1$ the rates become rapidly smaller as
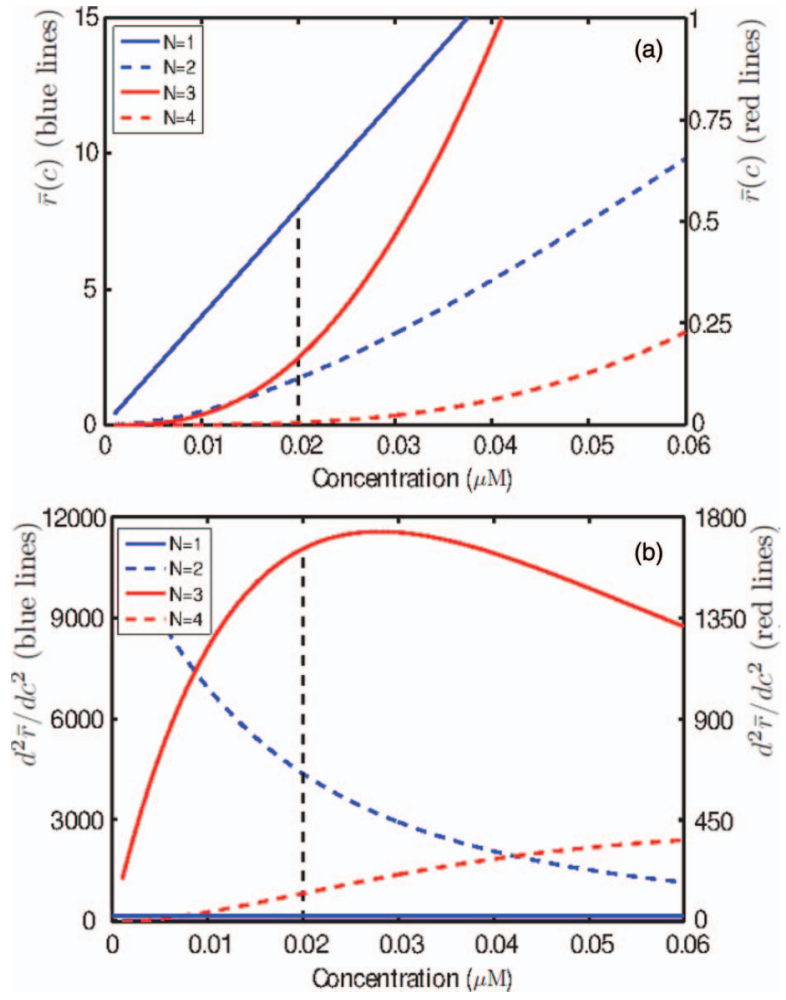

FIG. 4. (a) Channel opening rate estimate $\bar{r}$ given by (9) versus concentration for $N=1$ (blue solid line), $N=2$ (blue dashed line), $N=3$ (red solid line), and $N=4$ (red dashed line). (b) The $d^{2} \bar{r} / d c^{2}$ versus concentration for $N=1$ (blue solid line), $N=2$ (blue dashed line), $N=3$ (red solid line), and $N$ $=4$ (red dashed line) showing positive convexity for all concentrations. The dashed gray line indicates the approximate steady intracellular concentration (used in simulations) of $c_{0}=0.02 \mu \mathrm{M}$. All blue lines correspond with a $y$ axis on the left side of the plot and red lines correspond with a $y$ axis on the right side of the plot.

$N$ increases but these rates always have a convex relationship with concentration. These rates have been determined by (9) which approximates the rates that are expected as a result of MC simulations. It is therefore not surprising that the diffusive noise that is modelled in the TRM simulations reduces the expected time $\bar{\tau}$ compared with that of the non-spatial MC simulations. It is important to note, however, that this explanation for a possible noise-dependent change to the channel opening times is merely qualitative. This is because this noise fluctuates in time at rates that are dependent on the diffusion constant.

Figure 5 shows the comparison between the expected interpuff times $\tau$ of the non-spatial MC and spatial TRM simulations for variations in $N$. It shows that for $N=1$ there is no observable difference in $E(\tau)$ between the two numerical methods, as expected. There is a minor difference in the expected interpuff time for $N=2$, due to the fact that while the theoretical result (9) is a convex function of concentration for $N=2$, it is not sufficiently convex at small concentrations to observe large differences in the expected interpuff time. The increase in the curvature of (9) is more apparent when $N$ increases to 3 and 4 and the noise that is modelled in the concentration of ions in the TRM regime gives noticeably smaller interpuff times. The reason for the rapid increase in $E(\tau)$ as $N$ 


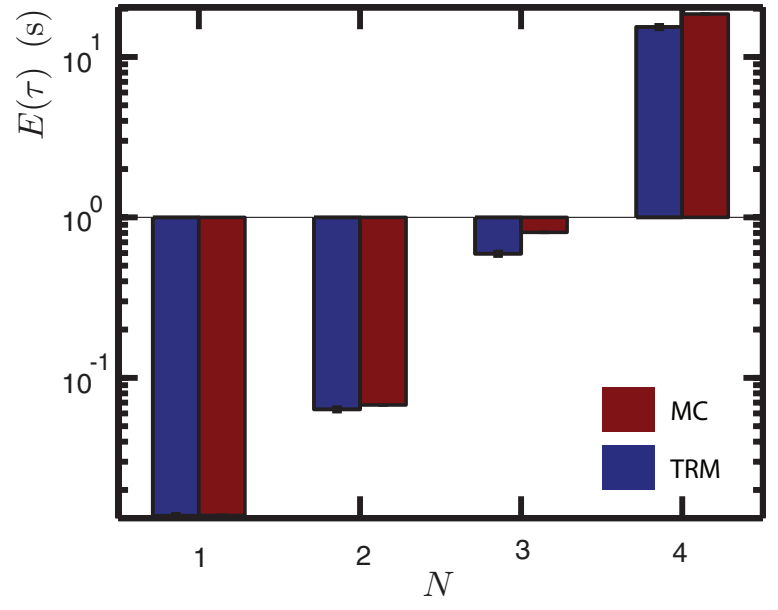

FIG. 5. Expected interpuff times $E(\tau)$ for variations in the required number of subunits $N$ that need to be active for a channel to open and initiate a puff measured using $10^{6} \mathrm{MC}$ simulations (red bars) and $10^{3}$ TRM simulations (blue bars). Parameters used: $C=9, k_{1}=100(\mu \mathrm{M} \mathrm{s})^{-1}, k_{2}=20 \mathrm{~s}^{-1}, c_{0}$ $=0.02 \mu \mathrm{M}$, and $D=220 \mu \mathrm{m}^{2} \mathrm{~s}^{-1}$ (for TRM simulations only).

increases is due to the significantly smaller probability $P_{\text {act }}$ when the ion concentration is $c_{0}=0.02 \mu \mathrm{M}$ (see (2)).

Using (9), we were able to make a heuristic argument for why expected interpuff times are reduced in the presence of diffusive noise. We did not consider the dynamic behavior of the channel opening rate $\bar{r}(t)$ due to temporal fluctuations resulting from the size of the diffusion constant. It was rather assumed that the system of channel states would react instantaneously to their average steady states given temporal fluctuations in concentration. It is therefore important to note how the temporal fluctuation in concentration may affect the interpuff time by looking at the expected interpuff times as a function of the diffusion constant. This relationship is shown in Figure 6 for interpuff times of a cluster of nine channels and a single channel. If diffusion is sufficiently large $D$ $\gtrsim 100 \mu \mathrm{m}^{2} \mathrm{~s}^{-1}$ then the expected interpuff time is relatively stable to changes in the diffusion constant and the expected

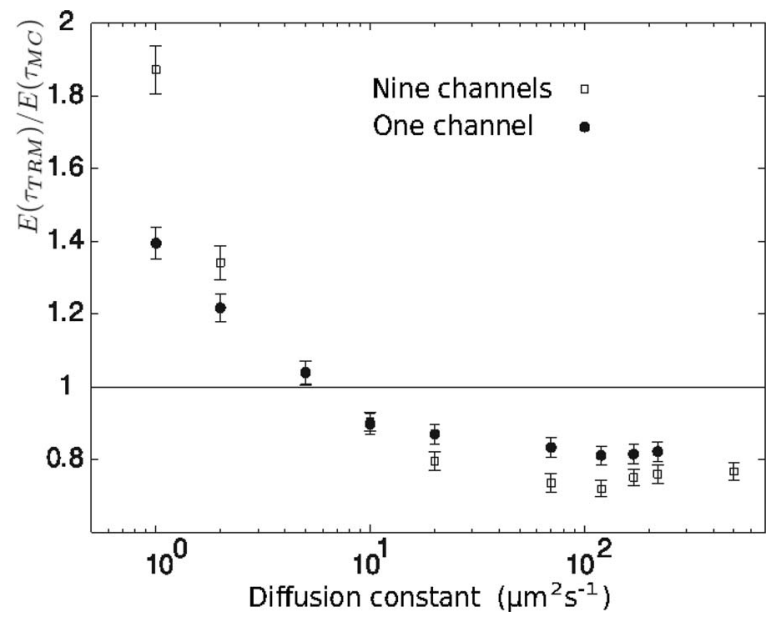

FIG. 6. The ratio of the expected interpuff times computed using the TRM and using the Monte Carlo channel state simulation for different values of the diffusion constant $D$ (found from $10^{3}$ TRM simulations for each value of $D$ ). The solid line indicates the ratio equal to 1 . Parameters used: $N=3, C=9$ (hollow squares), $C=1$ (filled circles), $k_{1}=100(\mu \mathrm{Ms})^{-1}, k_{2}=20 \mathrm{~s}^{-1}$, and $c_{0}=0.02 \mu \mathrm{M}$. interpuff time is less than that expected using a Monte Carlo simulation of the channel states and assuming constant rates of activation. We found this to be the case both for the cluster of nine channels and a single channel thereby excluding effects due to the coupling of these channels in the cluster. While it has been shown that receptor clustering affects the interpuff time, ${ }^{31}$ we have found these effects to be small compared to the effect of diffusive noise using our TRM model. Furthermore, all expected interpuff times were found to have exponential distributions. This means that our chosen initial conditions on the states of the channels are close to the quasisteady state of the system and that transient effects are not artificially reducing the expected time for a puff. If the diffusion constant is sufficiently small, the rate a channel opens is significantly reduced because the momentary activation of a subunit often leaves a relative void of ions to further activate other subunits. This void is filled slowly since diffusion is slow.

At higher values of the diffusion constant, the discrepancy between non-spatial MC simulation opening times and TRM simulation opening times are harder to explain. Let us define the copy number of candidate ions in some local region to the channels for reaction with a channel subunit as a noisy, discrete, function of time $\phi_{\mathcal{N}}(t)$. Here $\mathcal{N}$ represents the expected number of molecules that exist in this local region. Given a random snapshot of the value of $\phi_{\mathcal{N}}$, each ion has an equal probability to be at any region of space and can be considered independent of each other. Therefore $\phi_{\mathcal{N}}$ should be distributed according to a Poisson distribution with a mean of $\mathcal{N}$. The function $\phi_{\mathcal{N}}(t)$ spends some time above the mean and some time below the mean. While the function is above the mean the opening rate is increased more significantly than the amount that it is decreased when the function is below the mean. This is because an increase in the local concentration does not just increase the likelihood of a reaction between ions and channels but it also increases the likelihood that these channels are in the $\{N-1\}$ state (that is, they are one reaction away from opening). One might argue that if the temporal fluctuation of the function $\phi_{\mathcal{N}}(t)$ is great enough (which can be achieved by increasing the diffusion constant $D$ ) that there is not enough time for the system to react to a stochastic increase in the function. That is, the relaxation time that is needed to significantly increase the likelihood that the channels are in the $\{N-1\}$ state is longer than the time the function $\phi_{\mathcal{N}}(t)$ remains above $\mathcal{N}$ and therefore the rate of opening should be proportional to the instantaneous value of $\phi_{\mathcal{N}}(t)$. Given this argument, the discrepancy between MC simulation opening times and TRM simulation opening times cannot be explained heuristically as we have previously done. To show that this is not the case for large $D$ and indeed the source of the discrepancy between MC and TRM simulations is noise in the local concentration, consider our local domain to be the "region of interest," $\Omega_{B}$, in our TRM simulations. The expected number of molecules inside this region over the time of the simulation averaged over 4500 TRM simulations with diffusion constant $D=500 \mu \mathrm{m}^{2} \mathrm{~s}^{-1}$ (using a cluster $C$ $=9$ ) was measured to be $\mathcal{N}=12.046$ which corresponds to an average concentration of $c_{0}=0.02 \mu \mathrm{M}$. If the opening of channels was not dependent on the noise in the local 
concentration of calcium, then one would expect the number of ions in $\Omega_{B}$ to be equal to that calculated for the final critical reaction event that leads to the channels opening. In such a scenario, the expected number of molecules in $\Omega_{B}$ at the time of channel opening should be $\mathcal{N}+1=13.046$. This is computed by taking the average number of candidate ions in $\Omega_{B}$ conditional on the opening of a channel and using the fact that the probability of an opening event is proportional to the number of candidate ions and for an arbitrary moment in time this number of ions is distributed according to a Poisson distribution with mean of $\mathcal{N}=12.046$. However, the number of ions in $\Omega_{B}$ at the moment at which a channel opens was averaged over the 4500 simulations and found to be equal to 14.1 \pm 0.05 . This means that for a large diffusion constant $D$, the channels are more likely to open when the local concentration fluctuates above its expected value if the system were not influenced by noise.

\section{DISCUSSION}

In this paper we have tested a basic assumption of many studies on $\mathrm{Ca}^{2+}$ signaling, which is that the $\mathrm{Ca}^{2+}$ concentration, locally or globally defined, can be regarded as a deterministic quantity. We have asked whether the properties of collective release from channels change if $\mathrm{Ca}^{2+}$ ions are treated as discrete entities with Brownian motion trajectories. We have found that the time intervals between stochastically occurring $\mathrm{Ca}^{2+}$ puffs decrease by about $20 \%$. This result is obtained under standard assumptions on the gating dynamics of an $\mathrm{IP}_{3} \mathrm{R}$ channel and diffusion constant $D=220 \mu \mathrm{m}^{2} \mathrm{~s}^{-1}$.

The interpuff interval is here interpreted as a waiting time for a first-channel opening. The real IPI depends on other properties as well such as the refractory period after the termination of a puff. Those refractory periods have been studied recently and were found to be rather small. ${ }^{32}$ Comparing our finding with experimental values we see that in mammalian neuroblastoma cells Smith and Parker ${ }^{33}$ find periods of about $5 \mathrm{~s}$, much larger than in our estimates. Our results have clarified, that the effect of diffusive noise does not explain directly the deviations of non-spatial MC modeling and experiments. On the contrary, incorporation of discrete $\mathrm{Ca}^{2+}$ ions increases the deviation for large diffusion constant and makes the search for a possible mechanism explaining the disparities more urgent. For small diffusion constants, however, the interval increases strongly compared to the non-spatial result. It is interesting to note that a small $\mathrm{Ca}^{2+}$ diffusion constant may be obtained in cells with large concentrations of $\mathrm{Ca}^{2+}$ binding proteins. Here, binding of $\mathrm{Ca}^{2+}$ ions to the proteins reduces the effective $\mathrm{Ca}^{2+}$ diffusion constant in deterministic reaction-diffusion equations $\mathrm{s}^{34}$ and it remains to be studied if our result for small diffusion constant is relevant in this context. While this is interesting to note, these binding proteins are incapable of reducing the diffusion constant to the small values that are required by the TRM model to explain the experimental results of Smith and Parker. ${ }^{33}$ It remains unclear whether other factors can explain the larger experimental IPIs compared to our simulations.

Our results clearly contrast earlier studies that neglect noise from calcium ion discreteness. While many authors sim- ply assume that such deterministic modeling is valid, there is also evidence from numerical simulations supporting this approach. Hake and Lines, for instance, have concluded that $\mathrm{Ca}^{2+}$ dynamics in small cellular compartments is well described by deterministic $\mathrm{Ca}^{2+}$ diffusion and stochastic binding to receptors. ${ }^{35}$ Our problem differs from the setup analyzed by Hake and Lines since we consider a larger, practically cell-wide domain. A second, perhaps more significant difference to their study is that we consider a more complex channel gating model, i.e., we determine the waiting times until several ions have bound to the channel. Therefore, and although we here analyzed a strongly simplified model for calcium puffs, our result casts serious doubt on the usefulness of deterministic $\mathrm{Ca}^{2+}$ equations for more complex dynamical models of calcium signals.

The fundamental mechanism for decreased interpuff intervals due to the presence of $\mathrm{Ca}^{2+}$ diffusion is caused by the critical number of active subunits required for a puff. The number of active subunits has to be collected over time. This collection has to stochastically occur rapidly to reach the critical number of active subunits before the stochastic deactivation of the subunits stops the collection and forces the channel to start collecting again from beginning. At low diffusion rates, after each absorption of $\mathrm{Ca}^{2+}$, the lack of sufficient diffusion of surrounding $\mathrm{Ca}^{2+}$ decreases the collection rate and ultimately significantly increases the interpuff time. If this diffusion rate is sufficiently large there is a tendency for the spatial noise to decrease the interpuff time (compared to nonstochastic models) since the increase in the opening rate that is due to stochastically larger local concentrations is greater than the decrease in the opening rate due to stochastically lower local concentrations.

\section{SUMMARY}

In this paper, we have produced a simple model of $\mathrm{IP}_{3} \mathrm{R}$ calcium ion channels. In particular, we focused on the distribution and expected opening times for these channels. It was found that for realistically small concentrations of calcium in the cytosol, that the channels open at intervals which are approximately exponential in distribution. A simple expression for the expected time for the channel to open can be determined to within approximately $10 \%$ of that predicted by the simple model. The simple model was simulated using a Monte Carlo algorithm which determined state changes in the calcium occupancy of the ion channels using a Markov chain (5). This model was found to give incorrect channel opening times due to high levels of localised noise that is characteristic of the low concentrations of calcium ions within the cytosol. Specifically, for low calcium ion diffusion constants, channel opening times can be significantly increased due to noisy spatial distributions in the expected calcium ion distribution moments after a calcium ion is absorbed by an ion channel. For high calcium ion diffusion constants, channel opening times measured using a mean field approach like that of our Monte carlo simulations, may be incorrect by up to about 50\% of the true value due to the nonlinear relationship between the expected rate of channel opening and the effective local ion concentration (9) which fluctuates with diffusion. If the 
number of active subunits required to open the channel is reduced, this nonlinear relationship disappears and the simple model is valid. The spatial TRM model that was used in this manuscript demonstrates behavior that is not captured by the simple Monte Carlo model.

\section{ACKNOWLEDGMENTS}

The research leading to these results has received funding from the European Research Council under the European Community's Seventh Framework Programme (FP7/20072013) / ERC Grant Agreement No. 239870. This publication was based on the work supported in part by Award No. KUKC1-013-04, made by King Abdullah University of Science and Technology (KAUST). Radek Erban would also like to thank Somerville College, University of Oxford, for a Fulford Junior Research Fellowship; Brasenose College, University of Oxford, for a Nicholas Kurti Junior Fellowship; the Royal Society for a University Research Fellowship; and the Leverhulme Trust for a Philip Leverhulme Prize. This prize money was used to support research visits of Sten Rüdiger in Oxford. Sten Rüdiger also acknowledges support from the Deutsche Forschungsgemeinschaft.

${ }^{1}$ M. B. Flegg, S. J. Chapman, and R. Erban, "The two-regime method for optimizing stochastic reaction-diffusion simulations," J. R. Soc., Interface 9, 859-868 (2012).

${ }^{2}$ B. Alberts, A. Johnson, J. Lewis, M. Raff, K. Roberts, and P. Walter, Molecular Biology of the Cell (Garland Science, New York, 2007).

${ }^{3}$ A. Goldbeter, G. Dupont, and M. J. Berridge, "Minimal model for signalinduced $\mathrm{Ca}^{2+}$ oscillations and for their frequency encoding through protein phosphorylation," Proc. Natl. Acad. Sci. U.S.A. 87, 1461-1465 (1990).

${ }^{4}$ G. DeYoung and J. Keizer, "A single-pool inositol 1,4,5-trisphosphatereceptor-based model for agonist-stimulated oscillations in $\mathrm{Ca}^{2+}$ concentration," Proc. Natl. Acad. Sci. U.S.A. 89, 9895-9899 (1992).

${ }^{5}$ J. Yao, J. Choi, and I. Parker, "Quantal puffs of intracellular $\mathrm{Ca}^{2+}$ evoked by inositol trisphosphate in xenopus oocytes," J. Physiol. 482, 533 (1995).

${ }^{6}$ J. Foskett, C. White, K. Cheung, and D. J. Mak, "Trisphosphate receptor $\mathrm{Ca}^{2+}$ release channels," Physiol. Rev. 87, 593 (2007).

${ }^{7}$ G. Dupont, L. Combettes, and L. Leybaert, "Calcium dynamics: Spatiotemporal organization from the subcellular to the organ level," Int. Rev. Cytol. 261, 193-245 (2007).

${ }^{8} \mathrm{I}$. Smith and I. Parker, "Imaging the quantal substructure of single $\mathrm{IP}_{3} \mathrm{R}$ channel activity during $\mathrm{Ca}^{2+}$ puffs in intact mammalian cells," Proc. Natl. Acad. Sci. U.S.A. 106, 6404 (2009).

${ }^{9}$ C. Wei, X. Wang, M. Chen, K. Ouyang, L.-S. Song, and H. Cheng, "Calcium flickers steer cell migration," Nature (London) 457, 901-905 (2008).

${ }^{10}$ J. D. Capite, S. Ng, and A. Parekh, "Decoding of cytoplasmic $\mathrm{Ca}^{2+}$ oscillations through the spatial signature drives gene expression," Curr. Biol. 19, 853-858 (2009).

${ }^{11}$ A. Parekh, "Decoding cytosolic $\mathrm{Ca}^{2+}$ oscillations," Trends Biochem. Sci. 36, 78 (2010).

${ }^{12}$ S. Swillens, G. Dupont, and P. Champeil, "From calcium blips to calcium puffs: Theoretical analysis of the requirements for interchannel communication,” Proc. Natl. Acad. Sci. U.S.A. 96, 13750-13755 (1999).
${ }^{13}$ M. Falcke, "On the role of stochastic channel behavior in intracellular $\mathrm{Ca}^{2+}$ dynamics," Biophys. J. 84, 42-56 (2003).

${ }^{14}$ S. Rüdiger, C. Nagaiah, G. Warnecke, and J. W. Shuai, "Calcium domains around single and clustered $\mathrm{IP}_{3}$ receptors and their modulation by buffers," Biophys. J. 99, 3-12 (2010).

${ }^{15}$ J. Shuai and P. Jung, "Optimal ion channel clustering for intracellular calcium signaling," Proc. Natl. Acad. Sci. U.S.A. 100, 506-510 (2003).

${ }^{16} \mathrm{H}$. DeRemigio, J. Groff, and G. Smith, "Calcium release site ultrastructure and the dynamics of puffs and sparks," Math. Med. Biol. 25, 65 (2008).

${ }^{17}$ S. Rüdiger, J. Shuai, and I. Sokolov, "Law of mass action, detailed balance, and the modeling of calcium puffs," Phys. Rev. Lett. 105, 048103 (2010).

${ }^{18}$ H. Berg and E. Purcell, "Physics of chemoreception," Biophys. J. 20, 193219 (1977).

${ }^{19}$ W. Bialek and S. Setayeshgar, "Physical limits to biochemical signaling," Proc. Natl. Acad. Sci. U.S.A. 102, 10040-10045 (2005).

${ }^{20} \mathrm{~J}$. Elf and M. Ehrenberg, "Fast evaluation of fluctuations in biochemical networks with the linear noise approximation," Genome Res. 13, 24752484 (2003)

${ }^{21}$ I. Parker, J. Choi, and Y. Yao, "Elementary events of insp3-induced $\mathrm{Ca}^{2+}$ liberation in xenopus oocytes: Hot spots, puffs, and blips," Cell Calcium 20, 105-121 (1996).

${ }^{22}$ G. D. Dickinson, D. Swaminathan, and I. Parker, "The probability of triggering calcium puffs is linearly related to the number of inositol trisphosphate receptors in a cluster," Biophys. J. 102, 1826-1836 (2012).

${ }^{23} \mathrm{M}$. Gibson and J. Bruck, "Efficient exact stochastic simulation of chemical systems with many species and channels," J. Phys. Chem. A 104, 18761889 (2000).

${ }^{24}$ R. Erban, J. Chapman, and P. Maini, "A practical guide to stochastic simulations of reaction-diffusion processes," preprint arXiv:0704.1908 [qbio.SC] (2007).

${ }^{25}$ S. S. Andrews and D. Bray, "Stochastic simulation of chemical reactions with spatial resolution and single molecular detail," Phys. Biol. 1, 137-151 (2004).

${ }^{26}$ S. Engblom, L. Ferm, A. Hellander, and P. Lötstedt, "Simulation of stochastic reaction-diffusion processes on unstructured meshes," SIAM J. Sci. Comput. (USA) 31, 1774-1797 (2009).

${ }^{27}$ J. Lipkova, K. C. Zygalakis, S. J. Chapman, and R. Erban, "Analysis of brownian dynamics simulations of reversible biomolecular reactions," SIAM J. Appl. Math. 71, 714-730 (2011).

${ }^{28}$ R. Erban and S. J. Chapman, "Stochastic modelling of reaction-diffusion processes: algorithms for bimolecular reactions," Phys. Biol. 6, 046001 (2009).

${ }^{29}$ R. Erban and S. J. Chapman, "Reactive boundary conditions for stochastic simulations of reaction-diffusion processes," Phys. Biol. 4, 16-28 (2007).

${ }^{30}$ A. W. Bowman and A. Azzalini, Applied Smoothing Techniques of Data Analysis (Oxford University Press, Oxford, 1997).

${ }^{31}$ B. R. Caré and H. A. Soula, "Receptor clustering affects signal transduction at the membrane level in the reaction-limited regime," Phys. Rev. E 87, 012720 (2013)

${ }^{32}$ K. Thurley, I. Smith, S. Tovey, C. Taylor, I. Parker et al., "Timescales of $\mathrm{IP}_{3}$-evoked $\mathrm{Ca}^{2+}$ spikes emerge from $\mathrm{Ca}^{2+}$ puffs only at the cellular level," Biophys. J. 101, 2638-2644 (2011).

${ }^{33}$ I. F. Smith, S. M. Wiltgen, and I. Parker, "Localization of puff sites adjacent to the plasma membrane: Functional and spatial characterization of $\mathrm{Ca}^{2+}$ signaling in SH-SY5Y cells utilizing membrane-permeant caged $\mathrm{IP}_{3}$," Cell Calcium 45, 65-76 (2009).

${ }^{34}$ A. Sherman, G. D. Smith, L. Dai, and R. M. Miura, "Asymptotic analysis of buffered calcium diffusion near a point source," SIAM J. Appl. Math. 61, 1816-1838 (2001).

${ }^{35}$ J. Hake and G. Lines, "Stochastic Binding of $\mathrm{Ca}^{+}$Ions in the Dyadic Cleft; Continuous versus Random Walk Description of Diffusion," Biophys. J. 94, 4184 (2008). 